\title{
Energy and angular momentum of charged rotating black holes
}

\author{
J. M. Aguirregabiria, A. Chamorro and K. S. Virbhadra* \\ Dpto. de Física Teórica, Universidad del País Vasco, Apdo. 644, 48080 Bilbao, Spain
}

\begin{abstract}
We show that the pseudotensors of Einstein, Tolman, Landau and Lifshitz, Papapetrou, and Weinberg (ETLLPW) give the same distributions of energy, linear momentum and angular momentum, for any Kerr-Schild metric. This result generalizes a previous work by Gürses and Gürsey that dealt only with the pseudotensors of Einstein and Landau and Lifshitz. We compute these distributions for the Kerr-Newman and Bonnor-Vaidya metrics and find reasonable results. All calculations are performed without any approximation in Kerr-Schild Cartesian coordinates. For the Reissner-Nordström metric these definitions give the same result as the Penrose quasi-local mass. For the Kerr black hole the entire energy is confined to its interior whereas for the KerrNewman black hole, as expected, the energy is shared by its interior as well as exterior. The total energy and angular momentum of the Kerr-Newman black hole are $M$ and $M a$, respectively ( $M$ is the mass parameter and $a$ is the rotation parameter). The energy distribution for the Bonnor-Vaidya metric is the same as the Penrose quasi-local mass obtained by Tod.

04.70.Bw,04.20.Cv
\end{abstract}

Typeset using REVTEX

*Present adress: Tata Institute of Fundamental Research, Homi Bhabha Road, Bombay 400005, India. 


\section{INTRODUCTION}

The general theory of relativity is an excellent theory of space, time and gravitation and has been supported by experimental evidences with flying colors, but some of its features are not without difficulties. For instance, the subject of energy-momentum localization has been a problematic issue since the outset of this theory. Einstein investigated whether or not one can obtain a locally conserved energy-momentum tensor for the gravitational field plus the source (given by the right hand side of the Einstein equations). However, the locally conserved energy-momentum complex constructed by him is neither a tensor nor it is symmetric and therefore its physical interpretation was questioned by several physicists, notably by Weyl, Pauli, and Eddington (see reference [1]). Tolman [2] obtained a new energy-momentum pseudotensor which is again not symmetric. However, Landau and Lifshitz (LL) [3] succeeded in constructing a symmetric energy-momentum pseudotensor which can therefore be used to obtain the angular momentum of a general relativistic system. Nevertheless, to use the pseudotensors of Einstein, Tolman, or LL, one is restricted to quasi-Minkowskian coordinates. Møller [4], arguing that to single out a particular coordinate system is not satisfactory from the general relativistic point of view, constructed a new energy-momentum pseudotensor and claimed that with it one was not constrained to use asymptotically Minkowskian coordinates. The energy and energy current density components of the Møller pseudotensor transform as a four-vector density with respect to the group of purely spatial transformations. However, three years later, Møller observed a serious drawback of his prescription [5], i.e., the total energy-momentum vector of a closed physical system is not a Lorentz four-vector. Thus, Møller's attempt to give a coordinate-independent prescription for energy calculations failed and therefore we will not discuss Møller's pseudotensor any more in this paper. In fact, following the energy-momentum pseudotensor of Einstein, a plethora of definitions for energy, momentum, and angular momentum of a general relativistic system have been proposed by many authors (see [6] and references therein). Komar [7] gave a coordinate-independent definition for the energy. Using his prescription, 
Cohen and de Felice [8] calculated the effective mass of the Kerr-Newman (KN) metric. The Komar mass for the Reissner-Nordström (RN) metric is $E=M-Q^{2} / r(M$ and $Q$ are the mass and charge parameters, respectively), which is not in agreement with the linear theory. Moreover, Tamburino and Winicour [9] pointed out that the Komar definition is not adequate for radiating systems. Penrose [10] proposed a quasi-local definition of mass, momentum, and angular momentum in general relativity. Using the Penrose definition, Tod [11] calculated the quasi-local mass for several spacetimes. For the RN metric he found $E=M-Q^{2} /(2 r)$. He pointed out that as opposed to the Komar energy his result is in agreement with the linear theory. However, the Penrose definition has not succeeded to deal with the Kerr metric [12]. Bergqvist [13] considered seven different definitions of quasi-local mass and found that not any two of them give the same result for the RN and Kerr spacetimes. Despite these problems there has been considerable interest in this subject in recent years (see [14, 15] and references therein).

As the energy-momentum complexes of Einstein, Tolman, and LL are not tensors under general coordinate transformations, many physicists do not take them seriously as prescriptions for energy-momentum localization in general relativity. By contrast, the total energy, momentum, and angular momentum (in LL prescription) are accepted unanimously when calculations are carried out in quasi-Minkowskian coordinates. Lindquist, Schwartz, and Misner [16], using the LL pseudotensor, calculated the energy, momentum, and power output for the Vaidya metric and got the expected result. One of the present authors (Virbhadra, referred to as KSV hereafter) [17] showed that the pseudotensors of Einstein, Tolman, and LL (ETLL) give the same and reasonable energy distribution in the KN field when calculations are carried out in Kerr-Schild (KS) Cartesian coordinates. He also obtained the angular momentum distribution in the LL prescription. However, his calculations were limited up to the third order of the rotation parameter. Switching off the charge parameter he found that there is no energy associated with the exterior of the Kerr black hole. Though the investigations were limited up to the third order of the rotation parameter, he conjectured that one would get the same result for the Kerr metric if the calculations were carried 
out exactly. Cooperstock and Richardson [18] extended the energy calculations up to the seventh order of the rotation parameter and found that the pseudotensors of ETLL give the same energy distribution for the KN metric. Moreover, their result supported the conjecture of KSV that there is no energy associated with the exterior of the Kerr black hole. Later on KSV [19] showed that the pseudotensors of ETLL yield the same energy and energy current density components for the Vaidya metric.

Recently two of the present authors (Chamorro and KSV) 20 obtained the energy distribution in the Bonnor-Vaidya (BV) spacetime [21] in the prescriptions of Einstein and LL. Both definitions give the same result as the Penrose prescription [12]. They also obtained the energy current density components (and power output) for the same metric. Both (Einstein and LL) prescriptions give the same reasonable result. Despite these successes this subject required more study. For instance, there are other pseudotensors known in the literature and many more can be constructed (with the property of divergence-free relation), which could give different results for the KN, BV or other spacetimes. Moreover, the result known for the KN metric was limited up to the seventh order of the rotation parameter and it could be possible that different pseudotensors disagree if calculations were exactly performed. The aim of this paper is to clarify these questions. We consider two more wellknown (symmetric) energy-momentum pseudotensors, i.e., the pseudotensors of Papapetrou and Weinberg [22] and show that all these pseudotensors lead to the same result for the KN as well as the BV spacetimes when calculations are carried out in KS Cartesian coordinates. Weinberg, using his pseudotensor, calculated the total energy, momentum, and angular momentum of the Kerr metric. He carried out calculations at infinite radial distance and therefore his results do not bear on energy-momentum distributions.

Only recently has been brought to our attention that in an interesting paper Gürses and Gürsey [23] showed that the pseusotensors of Einstein and LL coincide for all Kerr-Schild metrics. In this paper we extend that result by showing that the five pseudotensors of ETLLPW coincide for any Kerr-Schild metric and, in consequence, give the same energy and energy current density components for the $\mathrm{KN}$ as well as BV spacetimes. The rest 
of the paper is organized as follows: Sec. II presents the energy-momentum pseudotensors of ETLLPW. In Sec. III] we show that the five pseudotensors coincide if KS Cartesian coordinates can be used. Sec. $\square$ gives the results for the energy, momentum, and angular momentum distributions of the KN metric in KS Cartesian coordinates. The energy, momentum, and energy current density components are also given. We present the results corresponding to the BV metric in Sec. V. Sec. VI discusses the results obtained in previous sections.

Conventions. We use geometrized units in which the speed of light in vacuum $c$ and the Newtonian gravitational constant $G$ are taken to be equal to 1 , the metric has signature +--- , and Latin (Greek) indices take values $0 \ldots 3(1 \ldots 3)$.

\section{ENERGY-MOMENTUM PSEUDOTENSORS}

The energy-momentum pseudotensors of ETLLPW are given below:

(a) The pseudotensor of Einstein is [4]

$$
\Theta_{i}^{k}=\frac{1}{16 \pi} H_{i}^{k l}{ }_{, l}
$$

where

$$
H_{i}^{k l}=-H_{i}^{l k}=\frac{g_{i n}}{\sqrt{-g}}\left[-g\left(g^{k n} g^{l m}-g^{l n} g^{k m}\right)\right]_{, m} .
$$

$\Theta_{0}{ }^{0}, \Theta_{\alpha}{ }^{0}$, and $\Theta_{0}{ }^{\alpha}$ are the energy, momentum, and energy current density components. $\Theta_{i}{ }^{k}$ satisfies the local conservation laws:

$$
\frac{\partial \Theta_{i}{ }^{k}}{\partial x^{k}}=0
$$

The energy and momentum are given by

$$
P_{i}=\iiint \Theta_{i}{ }^{0} d x^{1} d x^{2} d x^{3} .
$$

Using Gauss's theorem one can write 


$$
P_{i}=\frac{1}{16 \pi} \iint H_{i}^{0 \alpha} n_{\alpha} d S
$$

where $n_{\alpha}$ is the outward unit normal vector and $d S$ is the infinitesimal surface element.

(b) The pseudotensor of Tolman is [2]

$$
\mathcal{T}_{i}^{k}=\frac{1}{8 \pi} U_{i}^{k l}, l
$$

where

$$
U_{i}^{k l}=\sqrt{-g}\left[-g^{p k} V_{i p}^{l}+\frac{1}{2} g_{i}^{k} g^{p m} V_{p m}{ }^{l}\right]
$$

with

$$
V_{j k}^{i}=-\Gamma_{j k}^{i}+\frac{1}{2} g_{j}^{i} \Gamma_{m k}^{m}+\frac{1}{2} g_{k}^{i} \Gamma_{m j}^{m}
$$

$\mathcal{T}_{0}{ }^{0}, \mathcal{T}_{\alpha}{ }^{0}$, and $\mathcal{T}_{0}{ }^{\alpha}$ are the energy, momentum, and energy current density components. $\mathcal{T}_{i}^{k}$ satisfies the local conservation laws:

$$
\frac{\partial \mathcal{T}_{i}^{k}}{\partial x^{k}}=0
$$

The energy and momentum are given by

$$
P_{i}=\iiint \mathcal{T}_{i}^{0} d x^{1} d x^{2} d x^{3}
$$

For time-independent metrics one can write

$$
P_{i}=\frac{1}{8 \pi} \iint U_{i}^{0 \alpha} n_{\alpha} d S
$$

(c) The symmetric pseudotensor of Landau and Lifshitz is [3]

$$
L^{i k}=\frac{1}{16 \pi} \lambda_{, l m}^{i k l m}
$$

where

$$
\lambda^{i k l m}=-g\left(g^{i k} g^{l m}-g^{i l} g^{k m}\right) .
$$


$L^{00}$ and $L^{\alpha 0}$ are the energy and energy current (momentum) density components. $L^{i k}$ satisfies the local conservation laws:

$$
\frac{\partial L^{i k}}{\partial x^{k}}=0 .
$$

The energy and momentum are given by

$$
P^{i}=\iiint L^{i 0} d x^{1} d x^{2} d x^{3}
$$

and the angular momentum is given by

$$
J^{i k}=\iiint\left(x^{i} L^{0 k}-x^{k} L^{0 i}\right) d x^{1} d x^{2} d x^{3} .
$$

Using Gauss's theorem, the energy and momentum are

$$
P^{i}=\frac{1}{16 \pi} \iint \lambda^{i 0 \alpha m}{ }_{, m} n_{\alpha} d S
$$

and the physically interesting components of $J^{i k}$ are

$$
J^{\alpha \beta}=\frac{1}{16 \pi} \iint\left(x^{\alpha} \lambda^{\beta 0 \sigma m}{ }_{, m}-x^{\beta} \lambda^{\alpha 0 \sigma m}{ }_{, m}+\lambda^{\alpha 0 \sigma \beta}\right) n_{\sigma} d S .
$$

(d) The symmetric pseudotensor of Papapetrou is 22]

$$
\Sigma^{i k}=\frac{1}{16 \pi} N_{, l m}^{i k l m},
$$

where

$$
N^{i k l m}=\sqrt{-g}\left(g^{i k} \eta^{l m}-g^{i l} \eta^{k m}+g^{l m} \eta^{i k}-g^{l k} \eta^{i m}\right)
$$

with

$$
\eta^{i k}=\operatorname{diag}(1,-1,-1,-1) .
$$

$\Sigma^{00}$ and $\Sigma^{\alpha 0}$ are the energy, and energy current (momentum) density components. $\Sigma^{i k}$ satisfies the local conservation laws:

$$
\frac{\partial \Sigma^{i k}}{\partial x^{k}}=0
$$


The energy and momentum are given by

$$
P^{i}=\iiint \Sigma^{i 0} d x^{1} d x^{2} d x^{3}
$$

and the angular momentum is given by

$$
J^{i k}=\iiint\left(x^{i} \Sigma^{0 k}-x^{k} \Sigma^{0 i}\right) d x^{1} d x^{2} d x^{3} .
$$

For time-independent metrics, one has

$$
P^{i}=\frac{1}{16 \pi} \iint N_{, \beta}^{i 0 \alpha \beta} n_{\alpha} d S
$$

and the physically interesting components of the angular momentum are

$$
J^{\alpha \beta}=\frac{1}{16 \pi} \iint\left(x^{\alpha} N_{, \gamma}^{0 \beta \gamma \sigma}-x^{\beta} N_{, \gamma}^{0 \alpha \gamma \sigma}-N^{0 \beta \sigma \alpha}+N^{0 \alpha \sigma \beta}\right) n_{\sigma} d S .
$$

(e) The symmetric pseudotensor of Weinberg is 22]

$$
W^{i k}=\frac{1}{16 \pi} D_{, l}^{l i k}
$$

where

$$
D^{l i k}=\frac{\partial h_{a}^{a}}{\partial x_{l}} \eta^{i k}-\frac{\partial h_{a}^{a}}{\partial x_{i}} \eta^{l k}-\frac{\partial h^{a l}}{\partial x^{a}} \eta^{i k}+\frac{\partial h^{a i}}{\partial x^{a}} \eta^{l k}+\frac{\partial h^{l k}}{\partial x_{i}}-\frac{\partial h^{i k}}{\partial x_{l}}
$$

and

$$
h_{i k}=g_{i k}-\eta_{i k}
$$

$\eta_{i k}$ is the Minkowski metric. Indices on $h_{i k}$ or $\partial / \partial x_{i}$ are raised or lowered with help of $\eta$ 's. It is clear that

$$
D^{l i k}=-D^{i l k}
$$

$W^{00}$ and $W^{\alpha 0}$ are the energy and energy current (momentum) density components. $W^{i k}$ satisfies the local conservation laws:

$$
\frac{\partial W^{i k}}{\partial x^{k}}=0
$$


The energy and momentum are given by

$$
P^{i}=\iiint W^{i 0} d x^{1} d x^{2} d x^{3}
$$

and the angular momentum is given by

$$
J^{i k}=\iiint\left(x^{i} W^{0 k}-x^{k} W^{0 i}\right) d x^{1} d x^{2} d x^{3} .
$$

Using Gauss's theorem, one has

$$
P^{i}=\frac{1}{16 \pi} \iint D^{\alpha 0 i} n_{\alpha} d S
$$

and the physically interesting components of the angular momentum are

$$
J^{\alpha \beta}=\frac{1}{16 \pi} \iint\left(x^{\alpha} D^{\sigma 0 \beta}-x^{\beta} D^{\sigma 0 \alpha}+\eta^{\sigma \alpha} h^{0 \beta}-\eta^{\sigma \beta} h^{0 \alpha}\right) n_{\sigma} d S .
$$

\section{KERR-SCHILD METRICS}

In the following we shall consider the algebraically special metrics of Kerr-Schild which are given by

$$
g_{i k}=\eta_{i k}-2 V l_{i} l_{k}
$$

in terms of the scalar function $V$ and the null vector $l_{i}$ which satisfies the following properties:

$$
g_{i k} l^{i} l^{k}=\eta_{i k} l^{i} l^{k}=0, \quad l^{i} l_{k ; i}=l^{i} l_{k, i}=0 .
$$

For all these metrics one has $g=-1, l^{i}=g^{i k} l_{k}=\eta^{i k} l_{k}$ and the inverse metric is

$$
g^{i k}=\eta^{i k}+2 V l^{i} l^{k}
$$

Gürses and Gürsey [23] pointed out that for these metrics the pseudotensors of Einstein and LL coincide and are proportional to the Einstein tensor:

$$
\Theta_{i}{ }^{k}=\eta_{i j} L^{j k}=\frac{1}{8 \pi} G_{i}{ }^{k}
$$


In fact, by using the properties of Kerr-Schild metrics it is not difficult to prove that for these metrics the five pseusotensors of ETLLPW will coincide because one always have in KS coordinates:

$$
\Theta_{i}{ }^{k}=\mathcal{T}_{i}^{k}=\eta_{i j} L^{j k}=\frac{1}{8 \pi} G_{i}{ }^{k}, \quad L^{i k}=\Sigma^{i k}=W^{i k}=\frac{1}{16 \pi} \Lambda_{, l m}^{i k l m},
$$

where

$$
\Lambda^{i k p q}=2 V\left(\eta^{i k} l^{p} l^{q}+\eta^{p q} l^{i} l^{k}-\eta^{i p} l^{k} l^{q}-\eta^{k q} l^{i} l^{p}\right)
$$

In consequence, the energy and momentum are

$$
P^{i}=\frac{1}{16 \pi} \iint \Lambda_{, m}^{i 0 \alpha m} n_{\alpha} d S
$$

and the physically interesting components of $J^{i k}$ are

$$
J^{\alpha \beta}=\frac{1}{16 \pi} \iint\left(x^{\alpha} \Lambda_{, m}^{\beta 0 \sigma m}-x^{\beta} \Lambda_{, m}^{\alpha 0 \sigma m}+\Lambda^{\alpha 0 \sigma \beta}\right) n_{\sigma} d S .
$$

\section{THE KERR-NEWMAN METRIC}

The KN spacetime, characterized by mass, charge, and rotation parameters, in KS Cartesian coordinates is given by the line element in Eq. (36) with the following choices for $V$ and $l_{i}[24]$ :

$$
\begin{aligned}
V & =\frac{2 M \rho^{3}-Q^{2} \rho^{2}}{2\left(\rho^{4}+a^{2} z^{2}\right)} \\
l_{i} d x^{i} & =d t+\frac{z}{\rho} d z+\frac{\rho}{\rho^{2}+a^{2}}(x d x+y d y)-\frac{a}{\rho^{2}+a^{2}}(x d y-y d x),
\end{aligned}
$$

where $\rho$ is defined by the positive root of

$$
\frac{x^{2}+y^{2}}{\rho^{2}+a^{2}}+\frac{z^{2}}{\rho^{2}}=1 .
$$

$a=0$ and $Q=0$ in (44) and (45) give the RN and Kerr spacetimes, respectively. For each value of $\rho$ Eq. (46) defines a surface that can be parameterized by two angular coordinates, $(\theta, \varphi)$, as follows: 


$$
\begin{aligned}
& x=\sqrt{\rho^{2}+a^{2}} \sin \theta \cos \varphi, \\
& y=\sqrt{\rho^{2}+a^{2}} \sin \theta \sin \varphi \\
& z=\rho \cos \theta .
\end{aligned}
$$

The components of the outward unit normal vector over the surface given by (46) (with $\rho$ constant) are

$$
\begin{aligned}
& n_{x}=\frac{\rho}{\Upsilon} \sin \theta \cos \varphi, \\
& n_{y}=\frac{\rho}{\Upsilon} \sin \theta \sin \varphi \\
& n_{z}=\frac{\sqrt{\rho^{2}+a^{2}}}{\Upsilon} \cos \theta,
\end{aligned}
$$

and the infinitesimal surface element is

$$
d S=\sqrt{\rho^{2}+a^{2}} \Upsilon \sin \theta d \theta d \varphi
$$

where

$$
\Upsilon=\sqrt{\rho^{2}+a^{2} \cos ^{2} \theta}
$$

Now, we calculate the energy, momentum, and angular momentum for the KN metric in $\mathrm{KS}$ cartesian coordinates and use the results of the previous section. The intermediate mathematical expressions are very lengthy and therefore we give only the final results, which have been obtained and checked by means of two different computer algebra systems. The energy and momentum inside a surface given by (46) with constant $\rho$ in all the prescriptions of ETLLPW are

$$
\begin{aligned}
& E=M-\frac{Q^{2}}{4 \rho}\left[1+\frac{\left(a^{2}+\rho^{2}\right)}{a \rho} \arctan \left(\frac{a}{\rho}\right)\right], \\
& P_{1}=P_{2}=P_{3}=0 .
\end{aligned}
$$

The physically important components of the angular momentum in all the prescriptions of LLPW are 


$$
\begin{aligned}
& J^{12}=a\left\{M-\frac{Q^{2}}{4 \rho}\left[1-\frac{\rho^{2}}{a^{2}}+\frac{\left(a^{2}+\rho^{2}\right)^{2}}{a^{3} \rho} \arctan \left(\frac{a}{\rho}\right)\right]\right\}, \\
& J^{23}=J^{31}=0 .
\end{aligned}
$$

The total energy, momentum, and angular momentum ( $\rho$ approaching infinity in the above expressions) for the $\mathrm{KN}$ metric are $E=M, P_{1}=P_{2}=P_{3}=0, J^{12}=M a$ and $J^{23}=J^{31}=0$. The energy, momentum, and energy current density components of the ETLLPW pseudotensors for the KN metric are

$$
\begin{aligned}
& \Theta_{0}{ }^{0}=\mathcal{T}_{0}{ }^{0}=L^{00}=\Sigma^{00}=W^{00}=\left(\rho^{4}+2 a^{2} \rho^{2}-a^{2} z^{2}\right) A, \\
& \Theta_{0}{ }^{1}=-\Theta_{1}{ }^{0}=\mathcal{T}_{0}{ }^{1}=-\mathcal{T}_{1}{ }^{0}=L^{10}=\Sigma^{10}=W^{10}=-2 a y \rho^{2} A, \\
& \Theta_{0}{ }^{2}=-\Theta_{2}{ }^{0}=\mathcal{T}_{0}{ }^{2}=-\mathcal{T}_{2}{ }^{0}=L^{20}=\Sigma^{20}=W^{20}=2 a x \rho^{2} A, \\
& \Theta_{0}{ }^{3}=\Theta_{3}{ }^{0}=\mathcal{T}_{0}{ }^{3}=\mathcal{T}_{3}{ }^{0}=L^{30}=\Sigma^{30}=W^{30}=0,
\end{aligned}
$$

where

$$
A=\frac{Q^{2} \rho^{4}}{8 \pi\left(\rho^{4}+a^{2} z^{2}\right)^{3}} .
$$

For the Kerr metric $(Q=0)$ all the components given in (53) are zero.

\section{THE BONNOR-VAIDYA METRIC}

Two of the present authors (Chamorro and KSV) [20] considered the BV metric in the prescriptions of Einstein and LL and got the same and reasonable result for the energy distribution. They also got the same result for the energy current density components. In the light of Gürses and Gürsey's result [23] one sees the reason for the coincidences. The BV metric in KS Cartesian coordinates is given by the line element in Eq. (36) with the following choices for $V$ and $l_{i}$ :

$$
\begin{aligned}
V & =\frac{M(u)}{r}-\frac{Q^{2}(u)}{2 r^{2}}, \\
l_{i} d x^{i} & =d t-d r
\end{aligned}
$$


where $r=\sqrt{x^{2}+y^{2}+z^{2}}$. The mass and charge parameters, $M(u)$ and $Q(u)$, depend on the retarded time coordinate $u(u=t-r)$. The pseudotensors of Einstein and LL give the same result 20]:

$$
E=M(u)-\frac{Q^{2}(u)}{2 r}
$$

It is of interest to note that the Penrose definition also leads to the same result for the BV metric [12]. Next we give the energy, momentum, and energy current density components for the BV metric in the prescriptions of ETLLPW.

$$
\begin{aligned}
& \Theta_{0}{ }^{0}=\mathcal{T}_{0}^{0}=L^{00}=\Sigma^{00}=W^{00}=\frac{Q^{2}}{8 \pi r^{4}}+r \Delta, \\
& \Theta_{0}{ }^{1}=-\Theta_{1}{ }^{0}=\mathcal{T}_{0}{ }^{1}=-\mathcal{T}_{1}^{0}=L^{10}=\Sigma^{10}=W^{10}=x \Delta, \\
& \Theta_{0}{ }^{2}=-\Theta_{2}{ }^{0}=\mathcal{T}_{0}{ }^{2}=-\mathcal{T}_{2}{ }^{0}=L^{20}=\Sigma^{20}=W^{20}=y \Delta, \\
& \Theta_{0}{ }^{3}=-\Theta_{3}{ }^{0}=\mathcal{T}_{0}{ }^{3}=-\mathcal{T}_{3}{ }^{0}=L^{30}=\Sigma^{30}=W^{30}=z \Delta,
\end{aligned}
$$

where

$$
\Delta=\frac{Q \dot{Q}-r \dot{M}}{4 \pi r^{4}}
$$

The dot over $Q$ and $M$ stands for the derivative with respect to the retarded time coordinate $u$.

\section{DISCUSSION}

The subject of the energy-momentum localization in general relativity has been debated since the beginning of relativity and it still continues (for instance, see [25]). Bondi [25] argued that a non-localizable form of energy is inadmissible in relativity and so its location can in principle be found. Following the Einstein pseudotensor, a large number of coordinatedependent as well as coordinate independent definitions of energy, momentum, and angular momentum in general relativity have been given in the literature. There is no adequate coordinate-independent prescription for energy-momentum localization in general relativity. 
Bergqvist [13 investigated seven different definitions of energy and reported that no two definitions give the same result for the RN and Kerr spacetimes. The well known quasi-local definition for energy, momentum, and angular momentum given by Penrose, which gave reasonable result for several spacetimes, has not succeeded to handle the Kerr metric [12].

In the present paper we have obtained the energy and angular momentum for the KN metric for arbitrary values of the mass, charge, and rotation parameters. The pseudotensors of ETLLPW give the same and reasonable energy distribution. Again the symmetric pseudotensors of LLPW give the same and reasonable angular momentum distribution for this metric. They also give the same energy and energy current density components for the KN metric. For the KN black hole the energy is distributed by its interior as well as exterior whereas for the Kerr black hole the energy is confined to its interior. This proves a previous conjecture of KSV [17] and is compatible with Cooperstock's conjecture [15]. It is clear from (51) and (52) that the energy distribution is independent of the sign on the charge as well as rotation parameters whereas the direction of the angular momentum depends on the sign of the rotation parameter and is independent of the sign on the charge parameter. This is obviously a convincing result. The total energy and angular momentum ( $\rho$ approaching infinity in (51) and (52)) are $M$ and $M a$, respectively. For the RN metric $(a=0)$, one gets

$$
E=M-\frac{Q^{2}}{2 r}
$$

The definitions of Penrose as well as that of Hayward give the same result for the RN metric [11,26]. Also, for the BV metric the pseudotensors of ETLLPW give the same result (see (57)) as given by the Penrose definition [12].

Summarizing, the energy-momentum localization has been a longstanding "recalcitrant problem" in general relativity. Despite many painstaking efforts no adequate coordinateindependent definition is known. We have shown that several pseudotensors give the same and reasonable result for the $\mathrm{KN}$ as well as the BV spacetimes when calculations are carried out in KS Cartesian coordinates. Different pseudotensors giving the same results for local quantities (in KS Cartesian coordinates) does not seem to be accidental. It could be of 
interest to investigate this problem further.

\section{ACKNOWLEDGMENTS}

One of us (KSV) thanks the Basque Government and the Tata Institute of Fundamental Research for financial support and J. M. Aguirregabiria, A. Chamorro, I. Egusquiza, and M. Rivas for hospitality. This work was supported in part by the University of the Basque Country under contract UPV/EHU 172.310-EA034/94. We thank F. I. Cooperstock for pointing out that a previous version of this work could be placed in perspective by properly taking into account the work by Gürses and Gürsey [23]. We also thank R. Jackiw, J. Katz, A. Komar, and K. P. Tod for helpful correspondence. 


\section{REFERENCES}

[1] S. Chandrasekhar and V. Ferrari, Proc. R. Soc. London A435, 645 (1991).

[2] R. C. Tolman, Relativity, Thermodynamics and Cosmology (Oxford Univ. Press, London, (1934) p. 227.

[3] L. D. Landau and E. M. Lifshitz, The Classical Theory of Fields Pergamon Press, 1987 p. 280 .

[4] C. Møller, Ann. Phys. (NY) 4, 347 (1958).

[5] C. Møller, Ann. Phys. (NY) 12, 118 (1961); D. Kovacs, Gen. Relativ. Grav. 17, 927 (1985); J. Novotny, Gen. Relativ. Grav. 19, 1043 (1987).

[6] J. D. Brown and J. W. York, Jr., Phys. Rev. D47, 1407 (1993).

[7] A. Komar, Phys. Rev. 113, 934 (1959).

[8] J. M. Cohen and F. de Felice, J. Math. Phys. 25, 992 (1984).

[9] L. A. Tamburino and J. H. Winicour, Phys. Rev. 150, 1039 (1966).

[10] R. Penrose, Proc. R. Soc. London A381, 53 (1982).

[11] K. P. Tod, Proc. R. Soc. London A388, 457 (1983).

[12] K. P. Tod (private communication).

[13] G. Bergqvist, Class. Quantum Grav. 9, 1753 (1992).

[14] K. P. Tod, Class. Quantum Grav. 2, L65 (1985); Class. Quantum Grav. 4, 1457 (1987); F. J. Tipler, Class. Quantum Grav. 2, L99 (1985); D. Lynden-Bell and J. Katz, Mon. Not. R. Astr. Soc. 213, 21 (1985); W. T. Shaw, Class. Quantum Grav. 3, L77 (1986); V. Chellathurai and N. Dadhich, Class. Quantum Grav. 7, 361 (1990); J. Katz and A. Ori, Class. Quantum Grav. 7, 787 (1990); R. Bartnik, Phys. Rev. Lett. 62, 2346 (1990); N. Rosen and F. I. Cooperstock, Class. Quantum Grav. 9, 2657 (1992); 
N. Rosen and K. S. Virbhadra, Gen. Relativ. Grav. 25, 429 (1993); K. S. Virbhadra and J. C. Parikh, Phys. Lett. B317, 312 (1993); Phys. Lett. B331, 302 (1994); D. H. Bernstein and K. P. Tod, Phys. Rev. D49, 2808 (1994); N. Rosen, Gen. Relativ. Grav. 26, 319 (1994); F. de Felice, Y. Yu, and S. Coriasco, Gen. Relativ. Grav. 26, 813 (1994);

[15] F. I. Cooperstock, in Topics on Quantum Gravity and Beyond, Essays in honor of L. Witten on his retirement, edited by F. Mansouri and J. J. Scanio (World Scientific, Singapore, 1993); Gen. Relativ. Grav. 26, 323 (1994).

[16] R. W. Lindquist, R. A. Schwartz, and C. W. Misner, Phys. Rev. 137, B 1364 (1965).

[17] K. S. Virbhadra, Phys. Rev. D41, 1086 (1990); Phys. Rev. D42, 1066 (1990); Phys. Rev. D42, 2919 (1990).

[18] F. I. Cooperstock and S. A. Richardson, in Proc. 4th Canadian Conf. on General Relativity and Relativistic Astrophysics (World Scientific, Singapore, 1991). Using $E=\iiint T_{0}^{0} d x^{1} d x^{2} d x^{3}$ they obtained an exact expression for the energy distribution in the KN spacetime.

[19] K. S. Virbhadra, Pramana-J. Phys. 38, 31 (1992).

[20] A. Chamorro and K. S. Virbhadra, hep-th/9406148, Pramana-J. Phys. (submitted).

[21] W. B. Bonnor and P. C. Vaidya, Gen. Relativ. Grav. 1, 127 (1970).

[22] A. Papapetrou, Proc. R. Irish. Acad. A52, 11 (1948); S. N. Gupta, Phys. Rev. 96, 1683 (1954); S. Weinberg, Gravitation and Cosmology: Principles and Applications of General Theory of Relativity (John Wiley and Sons, Inc., New York, 1972) p. 165. Recently one more symmetric energy-momentum pseudotensor has been given: D. Bak, D. Cangemi, and R. Jackiw, Phys. Rev. D49, 5173 (1994).

[23] M. Gürses and F. Gürsey, J. Math. Phys. 16, 2385 (1975).

[24] G. C. Debney, R. P. Kerr, and A. Schild, J. Math. Phys. 10, 1842 (1969). 
[25] C. W. Misner, K. S. Thorne and J. A. Wheeler Gravitation (W. H. Freeman and Co, NY, 1973) p. 467 and 603; F. I. Cooperstock and R. S. Sarracino, J. Phys. A11, 877 (1978); H. Bondi, Proc. R. Soc. London A427, 249 (1990).

[26] S. A. Hayward, Phys. Rev. D49, 831 (1994). 